\title{
ON A CLASS OF VIRTUAL KNOTS WITH UNIT JONES POLYNOMIAL
}

\author{
DANIEL S. SILVER and SUSAN G. WILLIAMS \\ Department of Mathematics and Statistics, University of South Alabama \\ Mobile, AL 36688 USA \\ email: silver@jaguar1.usouthal.edu,swilliam@jaguar1.usouthal.edu
}

\begin{abstract}
A collection of nontrivial virtual knots with unit Jones polynomial, obtained by virtualizing a single crossing of a knot diagram, contains no classical knots.

Keywords: virtual knot, Jones polynomial.
\end{abstract}

\section{Introduction.}

Whether there exists a nontrivial knot with unit Jones polynomial, the same as the unknot, remains an open question. In [K00] L. Kauffman gave a method for constructing infinitely many virtual knots (see below) with unit Jones polynomial, and he asked whether any of them is equivalent to a classical knot. We provide a negative answer for a large collection of such knots.

Our proof, which is algebraic, exploits a feature of classical knot diagrams that is not shared by all virtual knot diagrams: in the Wirtinger presentation of the knot group obtained from the diagram, any one relator is a consequence of the others (see [SW00]). Kauffman and Dye [DK03] have employed a different approach, using bracket polynomials for knots in thickened surfaces, to obtain partial results about a larger collection of virtual knots containing the ones that we consider.

\section{The theorem.}

A knot in the usual sense (henceforth referred to as a classical knot) can be regarded as an equivalence class of planar knot diagrams. Two diagrams are equivalent if one can be obtained from the other by a sequence of Reidemeister moves. In 1997 Kauffman introduced virtual knot diagrams, allowing a new type of crossing, called a virtual crossing and indicated by a small circle surrounding the site. Kauffman extended the classical Reidemeister moves to allow certain deformations 
involving virtual crossings. He then defined a virtual knot to be an equivalence class of virtual diagrams. We refer the reader to [K97], [K99] for details. It is a remarkable feature of the theory that two classical knots are equivalent under generalized Reidemeister moves if and only if they are equivalent under the classical ones [GPV00]. In this sense, virtual knot theory is an extension of the classical theory.

Let $K$ be a nontrivial classical knot, and assume that $S$ is a subset of the crossings of $K$ such that the knot obtained by switching these crossings is trivial. Let $K^{v}$ denote virtual knot that is obtained from $k$ by switching the crossings of $S$ but also placing a virtual crossing before and after each of the switched crossings (see Figure 1). Kauffman refers to the change as virtualizing the crossing. As shown in [K01], $K^{v}$ is a nontrivial virtual knot with unit Jones polynomial.

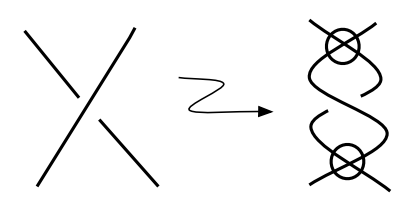

Figure 1: Virtualizing a crossing

The purpose of this note is to prove such virtual knots cannot be classical if $S$ consists of a single crossing.

Theorem 1. Assume that $K^{v}$ is a virtual knot constructed as above. If $|S|=1$, then $K^{v}$ is not classical.

Proof. We use the following result of W. Jaco and P.B. Shalen, a special case of Corollary 3.7 [JS76]. Let $\pi(k)$ be the group of a classical knot, and let $w$ be the class of any loop in the boundary of a tubular neighborhood of $k$. Then for any positive integer $n$, the centralizer of $w^{n}$ coincides with that of $w$.

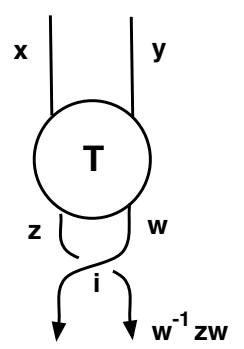

Figure 2: Tangle $T \cdot i$

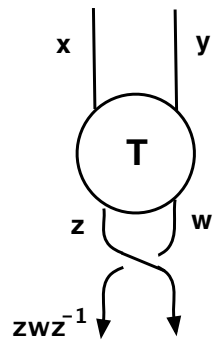

Figure 3: Switching crossing $i$

We can regard $K$ as the denominator closure of a tangle product $T \cdot i$, where the single crossing in $S$ comprises the tangle $i$. Label Wirtinger generators of the knot group $\pi(K)$ as in Figure 2. Then

$$
\pi(K) \cong \pi(T) /\left\langle\left\langle x=w, y=w^{-1} z w\right\rangle\right\rangle,
$$


where $\pi(T)$ is the group of the tangle, and $\langle\langle\cdots\rangle\rangle$ denotes normal closure. Since $K$ is classical, any Wirtinger relator is a consequence of the others; in particular, $y=$ $w^{-1} z w$ is a redundant relator in the presentation (1.1). By hypothesis, switching the crossing of $i$ converts $K$ to a trivial knot. Figure 3 is the corresponding diagram with labeled Wirtinger generators. We have

$$
\mathbf{Z} \cong \pi(T) /\left\langle\left\langle x=z w z^{-1}, y=z\right\rangle\right\rangle .
$$

Consider now the virtual knot $K^{v}$, the denominator closure of the tangle with labeled diagram in Figure 4 . We have

$$
\pi\left(K^{v}\right) \cong \pi(T) /\left\langle\left\langle x=w, y=w z w^{-1}\right\rangle\right\rangle .
$$

Since $y=w^{-1} z w$ is redundant in (1.1), it is a consequence of the relators of (1.3). Hence

$$
\begin{aligned}
\pi\left(K^{v}\right) & \cong \pi(T) /\left\langle\left\langle x=w, y=w z w^{-1}, y=w^{-1} z w\right\rangle\right\rangle \\
& \cong \pi(T) /\left\langle\left\langle x=w, y=w z w^{-1}, z w^{2}=w^{2} z\right\rangle\right\rangle .
\end{aligned}
$$

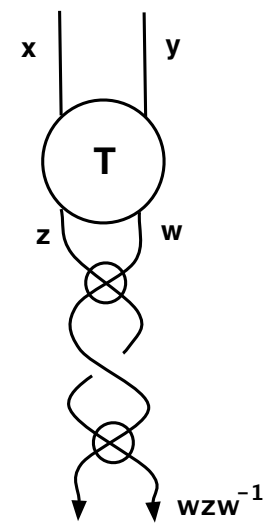

Figure 4: Virtualizing a crossing $i$

Assume that $K^{v}$ is a classical knot. Then some finite sequence of generalized Reidemeister moves will convert the denominator closure of the tangle in Figure 4 to a diagram $D$ that has only classical crossings. There is a corresponding sequence of Tietze moves on the associated group presentations, and is not difficult to see that the Wirtinger generator $w$ corresponds to a conjugate of Wirtinger generator of $D$. Since $D$ is classical, $w$ represents a meridian. Corollary 3.7 of [JS76] implies that the centralizer of $w^{2}$ is equal to that of $w$. Consequently, $z$ commutes with $w$ in $\pi\left(K^{v}\right)$, and we can write

$$
\begin{gathered}
\pi\left(K^{v}\right) \cong \pi(T) /\left\langle\left\langle x=w, y=w z w^{-1}, z w=w z\right\rangle\right\rangle \\
\cong \pi(T) /\left\langle\left\langle x=z w z^{-1}, y=z, z w=w z\right\rangle\right\rangle
\end{gathered}
$$


which in view of (1.2) is infinite cyclic.

We have shown that if $K^{v}$ is classical, then $\pi\left(K^{v}\right) \cong \mathbf{Z}$ and hence $K^{v}$ must be the unknot. However, by [K01] the involutory quandle $I\left(K^{v}\right)$ of $K^{v}$ is equal to that of $K$. Since $I(K)$ is nontrivial [W84], we have arrived at a contradiction. Hence $K^{v}$ is not classical.

\section{Acknowledgements}

The authors are grateful to Louis Kauffman for inspiration and helpful discussions. We also thank Wilbur Whitten pointing out the result of Jaco and Shalen used in the proof of Theorem 1.

Both authors were partly supported by National Science Foundation grant DMS0071004 .

\section{References}

[GPV00] M. Goussarov, M. Polyak and O. Viro, Finite type invariants of classical and virtual knots, Topology 39 (2000), 1045-1068.

[JS76] W. Jaco and P.B. Shalen, Peripheral structure of 3-manfifolds, Inventiones math. 38 (1976), 55-87.

[K97] L.H. Kauffman, Talks at MSRI meeting in January 1997, AMS meeting at University of Maryland, College Park in March 1997, Isaac Newton Institute Lecture in November 1997, Knots in Hellas Meeting in Delphi, Greece in July 1998, APCTP-NANKAI Symposium on Yang-Baxter Systems, Non-Linear Models and Applications at Seoul, Korea in October 1998.

[K99] L.H. Kauffman, Virtual knot theory, European J. Comb. 20 (1999) 663-690.

[K01] L.H. Kauffman, Detecting virtual knots, Atti Sem. Mat. Fis. Univ. Modena, Supplemento al Vol IL (2001), 241-282.

[DK03] H. Dye and L. H. Kauffman, Minimal surface representations of virtual knots and links. (in preparation).

[SW00] D.S. Silver and S.G. Williams, Virtual knot groups, in Knots in Hellas '98, Proceedings of the International Conference on Knot Theory and its Ramifications (C. McA. Gordon, V.F.R. Jones, L.H. Kauffman, L. Lambropoulou and J.H. Przytycki, eds) World Scientific, Singapore, 2000, 440-451.

[W84] S. Winker, Quandles knot invariants and the $N$-fold branched cover, Ph.D. thesis, Univ. of IL at Chicago, 1984. 